\title{
Efectos de la aplicación de programas de estiramientos sobre la musculatura isquiosural en escolares: revisión bibliográfica
}

Effects of stretching programs on hamstring flexibility

in schoolchildren: Literature review

\author{
Aida Mula \\ Departamento de Actividad Física y Deporte, Facultad de Ciencias del Deporte, Universidad de Murcia, España \\ Pilar Sainz de Baranda (D) \\ Departamento de Actividad Física y Deporte, Facultad de Ciencias del Deporte, Universidad de Murcia, España. \\ psainzdebaranda@um.es
}

\begin{abstract}
Resumen
La cortedad isquiosural es frecuente entre la población escolar. Por ello, es necesario desarrollar programas de intervención que incluyan estiramientos de la musculatura isquiosural desde las edades más tempranas. El objetivo del presente trabajo es analizar las características y los resultados de los programas de educación postural desarrollados dentro del contexto escolar con el objetivo de mejorar la extensibilidad isquiosural. La estrategia de búsqueda y selección de artículos empleada en esta revisión obtuvo un total de 171 artículos cuando se utilizaron las palabras clave "Hamstring and Program". Finalmente se analizaron 11 estudios que cumplían con los criterios de inclusión. Se analizaron diferentes variables entre las que destacan la duración de los programas, el tiempo de estiramiento y la técnica utilizada. Los resultados indican que la duración de los programas varía entre 8 y 32 semanas y el tiempo de estiramientos oscila entre 1 y 8 minutos. La mayoría de los estudios emplean la técnica de estiramientos estática pasiva y evalúan la extensibilidad isquiosural mediante el test dedos planta. Los resultados muestran una mejora significativa de la extensibilidad isquiosural tras la aplicación de los programas de estiramientos. Por ello, todos los estudios destacan el papel preventivo que puede jugar la Educación Física cuando se incluyen y desarrollan estiramientos específicos de la musculatura isquiosural como contenido de la materia.
\end{abstract}

Palabras claves: educación postural, estiramientos, isquiosurales, escolares.

\begin{abstract}
Short hamstrings are common among children in their school years. That is why it is needed to develop intervention programs including stretching of hamstring musculature from early ages. The goal of this paper is to analyze the characteristics and results of those programs aimed at postural education developed in the school context with the aim of improving hamstring flexibility. For the search and selection strategy of articles they key words 'hamstring and program' were used. After the research 171 articles were obtained but only 11 of these articles respected the criteria of this paper. Different variables have been analyzed, out of these variables the duration of the program, the time devoted to stretch and the technique used should be highlighted. Results show that the duration of the programs varies between 8 to 32 weeks and the time devoted to stretch varies between 1 and 8 minutes. Most of the studies use a passive technique for stretching and they measure hamstring flexibility through the toe-touch test. Research suggests a significant improvement of the hamstring flexibility after the application of stretching programs. As a consequence, all the studies highlight the preventive role of PE when it includes specific stretching of hamstring flexibility as part of the subject content.
\end{abstract}

Keywords: ural education, stretching, hamstrings, schoolchildren.

Recibido: 6-nov-2019

Aceptado: 12-nov-2019

Publicado: 20-nov-2019

Como citar (apa): Mula, A. y Sainz de Baranda, P. (2020). Efectos de la aplicación de programas de estiramientos sobre la musculatura isquiosural en escolares: Revisión bibliográfica . JUMP, (1), 53-66.

Como citar (vancouver): Mula, A. y Sainz de Baranda, P. Efectos de la aplicación de programas de estiramientos sobre la musculatura isquiosural en escolares: Revisión bibliográfica . JUMP. 2020;(1), 53-66.

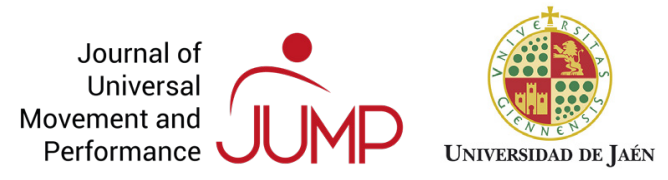




\section{Introducción}

En los últimos años la salud del escolar ha sido objeto de atención en la comunidad científica, sobre todo en lo que respecta a los cambios posturales de la columna vertebral y al dolor de espalda en niños y adolescentes (Martínez-García, 2013).

Diferentes estudios han tratado de identificar los factores de riesgo que se asocian al aumento de del dolor de espalda y las desalineaciones de la columna vertebral.

Con relación al dolor de espalda, Cardón y Balagué (2004) destacan 10 factores de riesgo que han sido identificados en diferentes investigaciones: el Índice de Masa Corporal (IMC) (Grimmer, Dansie, Milanese, Pirunsan y Trott, 2002), el sedentarismo (Groll, Heine, Goldammer y Zalpour, 2009), la prolongada sedentación (GarcíaAlen, 2009), trabajar fuera del horario escolar (Feldman et al., 2002; Harreby et al., 1999; Jones et al., 2003), la fuerza muscular (Sjölie y Ljunggren 2001), las mochilas (Moore, White y Moore, 2007), la flexibilidad de la musculatura (Jones et al., 2005), el tabaco (Balagué et al., 1999) y los factores psicosociales negativos (Cardón y Balagué, 2004).

En cuanto a las desalineaciones de la columna vertebral, Andújar (2010) resalta el aumento de la prevalencia de estás sobre todo en el plano sagital. Entre los factores que indica como justificación a este aumento destacan: la mala higiene postural, el desconocimiento en materia de higiene postural, el abuso de la sedentación y la cortedad de la musculatura isquiosural.

Respecto a la cortedad de la musculatura isquiosural, Santonja, Contreras y Ferrer (1995) la sitúan como la alteración más frecuente del raquis, con un porcentaje de prevalencia del $24,9 \%$ respecto a otras alteraciones como la hipercifosis $(6,4 \%)$, la hiperlordosis $(18,17 \%)$ o la escoliosis estructurada (9,3\%).

La importancia de la cortedad de la musculatura isquiosural es tanto por la prevalencia como por las repercusiones que puede ocasionar sobre la columna vertebral. De tal forma que la cortedad isquiosural limita el movimiento de la pelvis $\mathrm{y}$ ocasiona una mayor flexión del raquis dorsal y/o lumbar (Sainz de Baranda, Rodríguez, Santonja y Andujar, 2006).

Ferrer (1998) indica que la repetición y mantenimiento de este movimiento incorrecto podrá ocasionar:
- Acuñamientos vertebrales anteriores. Fundamentalmente debidos a retrasos en el crecimiento en la porción anterior de los cuerpos vertebrales, cuando estas presiones se produzcan y persistan en el momento crítico de su osificación. - Inversión de los espacios discales. Que podrán ser responsables de hernias lumbares de localización más atípica (T12 L4), cuando las inversiones sean marcadas y se acompañe de una probable "debilidad" ligamentosa.

- Hipercifosis dorsal. Cuando el patrón de movimiento del niño sea con hiperflexión del raquis dorsal, la cortedad isquiosural será un factor más de agravamiento de su cifosis dorsal, pero no su principal causa.

- Cifosis lumbar.

- Lumbalgias. Probablemente ocasionadas por las cadenas musculares junto a una incorrecta disposición mecánica.

Para evitar las consecuencias que conlleva la cortedad isquiosural sería conveniente incidir en el escolar por dos motivos. El primero de ellos, debido a que la columna vertebral del escolar se encuentra en un momento de crecimiento de alta vulnerabilidad (Santonja, Rodríguez, Saiz de Baranda y López-Miñarro, 2004). De tal forma que el crecimiento constante y las distintas etapas evolutivas por las que el niño pasa hacen que se puedan introducir patrones erróneos en su esquema en cualquier fase de la vida (Andújar y Santonja, 1996). El segundo motivo, hace referencia a que el escolar se encuentra en un entorno de aprendizaje (el centro escolar) que facilita la intervención en materia de higiene postural, la adquisición de conocimientos y de un estilo de vida saludable.

Estudios previos, indican la importancia de realizar programas preventivos en la etapa escolar, que ayuden a conseguir un crecimiento armónico de la columna vertebral y a disminuir los datos de prevalencia del dolor de espalda (Sainz de Baranda et al., 2006).

En esta línea, algunos programas de Educación Postural han sido desarrollados y evaluados en escolares. Las intervenciones desarrolladas han variado en múltiples aspectos, como la estructura y los contenidos del programa de intervención, su duración, la evaluación de los contenidos, la muestra utilizada o el profesional responsable del programa (Martínez, Gómez e Hidalgo, 2008).

Los resultadosindicanqueelabordajepreventivo supone una disminución de la prevalencia del 
dolor de espalda (Wedderkopp, Kjaer, Hestbaek, Korsholm y Leboeuf-Y, 2009), así como un aumento en la adquisición de conocimientos y una mejora en los hábitos posturales correctos que favorecen el cuidado de la espalda en niños y adolescentes (Sheldon, 1994; Cardon et al., 2000; Méndez y Gómez-Conesa, 2001; Geldhof, Cardon, De Bourdeaudhuij y De Clercq, 2007; Vidal, Borrás, Cantallops, Ponseti y Palou, 2010).

La revisión bibliográfica realizada por Rivas (2015) sobre programas de Educación Postural desarrollados en el contexto escolar, muestra como la gran mayoría de los programas se plantean como objetivo la mejora de los conocimientos conceptuales sobre el cuidado de la espalda y específicamente sobre la higiene postural (60\% de los estudios), seguido de los programas que buscan una mejora procedimental de la higiene postural (42\% de los estudios) y de aquellos que tras una intervención buscan una mejora en el morfotipo raquídeo (17\%) o de la condición física relacionada con la salud de la espalda (flexibilidad y/o resistencia de la musculatura del tronco) (14\%).

El objetivo de la presente revisión bibliográfica es analizar las características y los resultados de los programas de educación postural desarrollados dentro del contexto escolar con el objetivo de mejorar la extensibilidad isquiosural.

\section{Método}

\section{Criterios de Inclusión y Exclusión}

Para la selección de los estudios científicos se utilizaron los siguientes criterios de inclusión: (a) estudios experimentales (diseños controlados y aleatorios) y cuasi-experimentales (por ejemplo: pre-test/post-test), (b) ser un programa de educación postural que incluya o no estiramientos de la musculatura isquiosural, (c) en el caso de que el estudio midiese la extensibilidad isquiosural las pruebas de valoración debían ser lineales o angulares (rango de movimiento articular), (d) estudios que se fuesen realizados dentro del contexto escolar, (e) edad de los escolares comprendida entre los 5 y los 18 años.

Por otro lado, como criterios de exclusión se establecieron: (a) estudios científicos publicados en forma de resumen, comunicaciones cortas y/o cuyos datos no estuviesen publicados, (b) aquellos que no estuviesen redactados en inglés o en español, (c) estudios que incluyesen participantes con patologías en la columna vertebral, (d) estudios científicos no originales (e.g. cartas, comentarios, editoriales e informes de capítulos de libro sobre estudios publicados anteriormente) y (e) estudios que no explicasen y detallasen el método.

En la tabla 1 se muestra una descripción esquematizada de todos los criterios de inclusión y exclusión anteriormente expuestos.

\section{Estrategia de Búsqueda Bibliográfica}

La localización de artículos se realizó en las siguientes bases de datos informatizadas on-line:

- Pubmed. (http://www.ncbi.nlm.nih.gov/ pubmed): bases de datos de la Librería Nacional de Medicina de los Estados Unidos de América y de los Institutos Nacionales de Salud.

- $\quad$ Dialnet. (http://dialnet.unirioja.es): base de datos perteneciente a la Universidad de la Rioja. Es una de las mayores bases de datos de contenidos científicos en lenguas iberoamericanas y cuenta con diversos recursos documentales: artículos

Tabla 1. Criterios empleados para determinar la elegibilidad de los estudios científicos

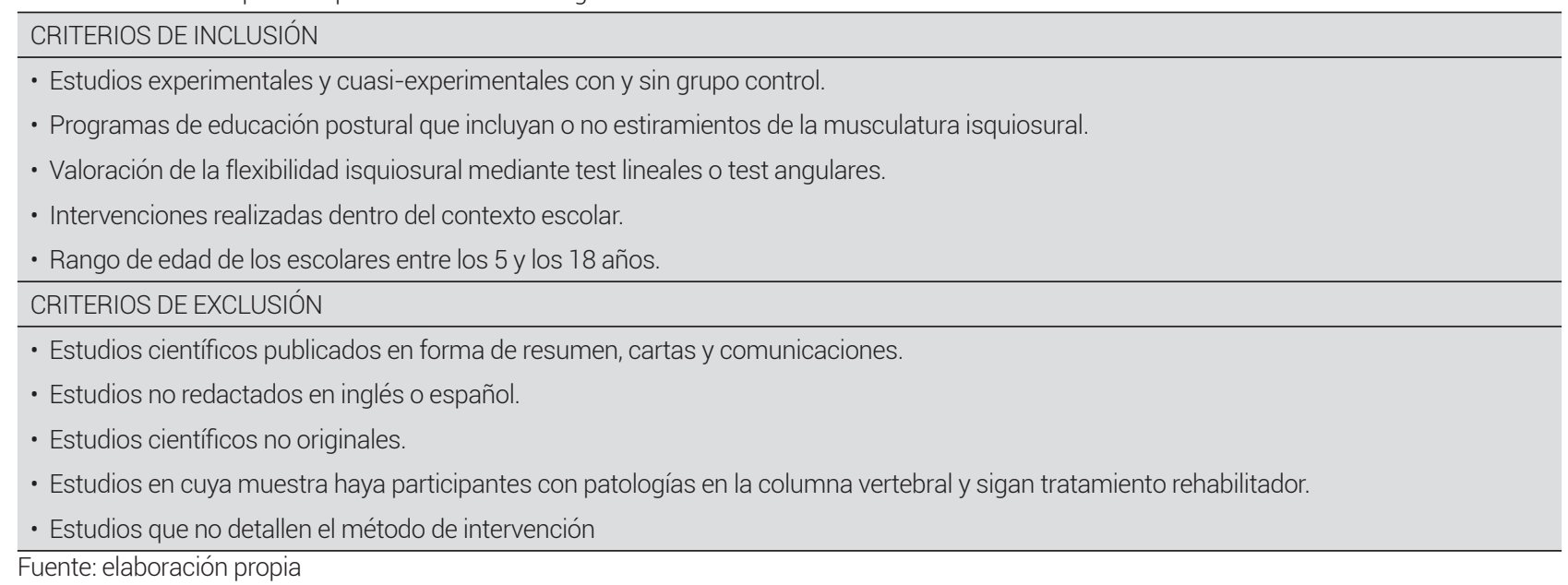


de revistas, artículos de obras colectivas, libros, actas de Congresos, reseñas bibliográficas y tesis doctorales.

- Google Scholar. (http://scholar.google.es): buscador de Google especializado en artículos de revistas científicas, enfocado por una base de datos disponible libremente en Internet, que almacena un amplio conjunto de trabajos de investigación científica de distintas disciplinas y en distintos formatos de publicación.

- Web of Science. (http://apps. webofknowledge.com): servicio en línea de información científica, suministrado por Thompson Reuters, integrado en ISI Web of Knowledge. Facilita el acceso a diferentes bases de datos en las que aparecen citas de artículos de revistas científicas, libros y otros tipos de material impreso que abarca todos los campos del conocimiento académico.

Las palabras "hamstrings" y "schoolchildren" fueron utilizadas siempre como criterio de búsqueda, de forma que, en las diversas exploraciones bibliográficas efectuadas, los términos "hamstrings" o "schoolchildren" estuvieron siempre presentes en uno de los campos de búsqueda, quedando el resto de campos subordinados con la preposición "and" y completos por una de las siguientes palabras: intervention, program, flexibility, tightness, back pain, postural education, postural hygiene, spine. La búsqueda finalizó el mayo de 2015.

Una vez seleccionados todos los artículos, cuyos títulos y resúmenes cumplían los criterios de inclusión, se procedió a su descarga en versión electrónica y posteriormente en versión impresa.

\section{Resultados}

\section{Selección de Estudios}

La estrategia de búsqueda y selección de artículos empleada en esta revisión obtuvo un total de 171 artículos cuando se utilizaron las palabras clave "hamstrings and schoolchildren". De todos los títulos y resúmenes seleccionados 34 cumplían los criterios de inclusión de realizarse en el contexto escolar y desarrollar un programa de educación postural. De los 34 estudios, 12 tuvieron un acceso restringido y/o fue imposible obtenerlos en formato electrónico, quedando un total de 22 artículos completos. De los 22 artículos obtenidos, 8 no cumplían el criterio de inclusión de desarrollar un programa de estiramientos específicos para la musculatura isquiosural, por lo que fueron excluidos para el análisis posterior. Por tanto, quedaron para el análisis un total de 11 artículos (figura 1).

Figura 1. Diagrama de la temporalización de la fase de selección de estudios científicos sobre los programas de educación postural en el contexto escolar con el objetivo de mejorar la flexibilidad isquiosural.

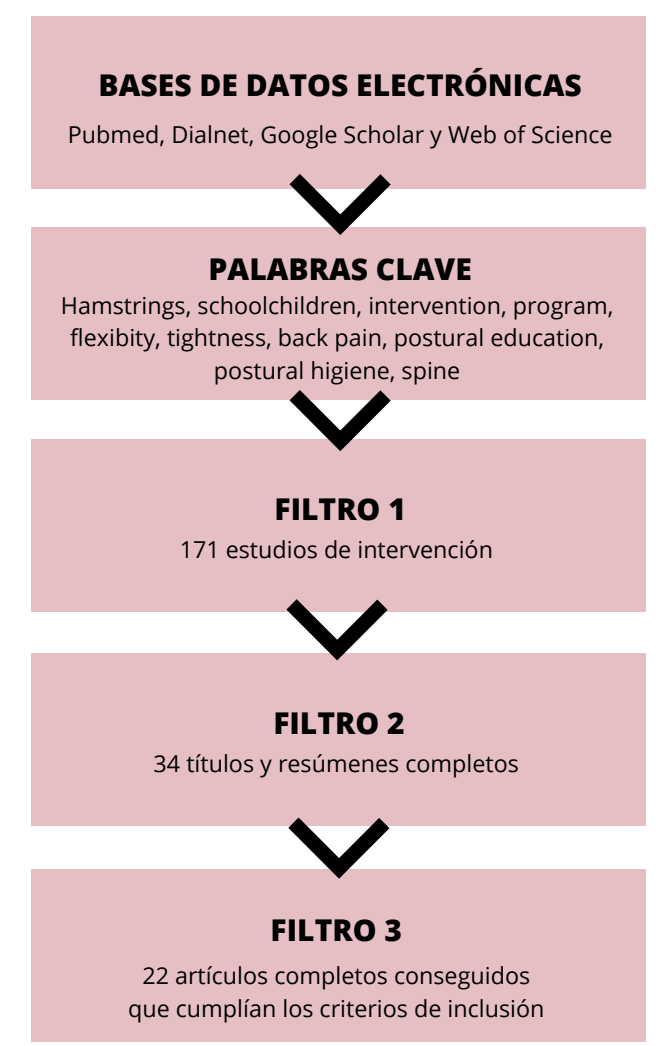

\section{Diseño de los estudios}

La tabla 2 muestra el número y porcentaje de estudios que utilizan cada uno de los diseños experimentales o cuasi-experimentales más habituales para evaluar la eficacia de un programa de intervención. Destaca el diseño de pre-test y pos-test con grupo control como el más empleado por los diversos autores con un $81,81 \%$, seguido del diseño con medidas repetidas con un 18,18\%.

\section{Población}

El total de escolares incluidos en los 11 estudios es de 853. En 8 trabajos señalan el número y las características de los escolares en función del sexo (Coledam, Aires de Arruda y Ramos de Olivera, 2012; 
Martínez-García, 2013; Mayorga-Vega, MerinoMarban, Garrido y Viciana, 2014; Rodríguez-García, Santonja, López-Miñarro y Sainz de Branda, 2008; Sainz de Baranda et al., 2006; Sainz de Baranda 2009; Sainz de Baranda, Rodríguez y Santonja, 2010; Sánchez-Rivas, Mayorga-Vega, Fernández y Merino-Marban, 2014), mientras que en los otros 3 no se especifican estos datos en función del sexo (Merino-Marban, Mayorga-Vega, Fernández, Estrada y Viciana, 2015; Rodríguez-García, LópezMiñarro y Alacid, 2013; Santonja, Sainz de Baranda, Rodríguez, López y Canteras, 2007).

Respecto a la edad, 6 estudios dan un dato general de la edad media de la muestra (MartínezGarcía, 2013; Mayorga-Vega et al., 2014; MerinoMarban et al., 2015; Sainz de Baranda, 2009; Sánchez-Rivas et al., 2014; Santonja et al., 2007), mientras que 2 estudios diferencian la media de la

Tabla 2. Diseño de los estudios científicos seleccionados

\begin{tabular}{lcc}
\hline Tipo de diseño & $\begin{array}{c}\text { Número de } \\
\text { estudios (n) }\end{array}$ & $\begin{array}{c}\text { Porcentaje de } \\
\text { estudios (\%) }\end{array}$ \\
\hline $\begin{array}{l}\text { Medidas repetidas } \\
\text { Pre-test y pos-test con grupo } \\
\text { control }\end{array}$ & 2 & $18,18 \%$ \\
$\begin{array}{l}\text { Pre-test y pos-test sin grupo } \\
\text { control }\end{array}$ & 0 & $81,81 \%$ \\
\hline
\end{tabular}

Fuente: elaboración propia muestra en función de los grupos (Sainz de Baranda et al., 2010; Santonja et al., 2007). El estudio de Rodríguez-García et al. (2008), Rodríguez-García et al. (2013) y Santonja et al. (2007) diferencian la edad media entre grupos de Educación Primaria y/o Secundaria; y Sainz de Baranda et al. (2006) entre el grupo control y el grupo experimental. Solo hay un estudio que diferencia la media de edad entre niños y niñas (Coledam et al., 2012).

Estudios de Educación Postural que desarrollan un programa de estiramientos dentro del contexto escolar

Una vez analizados los programas de intervención de los estudios seleccionados se resaltan varios aspectos.

Con relación a la duración de los programas, se pueden dividir los estudios en aquellos que emplean una duración entre las 8 y las 16 semanas (Coledam et al., 2012; Mayorga-Vega et al., 2014; Merino-Marban et al., 2015; Sánchez-Rivas et al., 2014), y aquellos que desarrollan el programa de estiramientos durante todo el curso académico, con una duración entre las 30 y las 32 semanas (Martínez-García, 2013; Rodríguez-García et al., 2008; Rodríguez-García et al., 2013; Sainz de Baranda et al., 2006; Sainz de Baranda 2009; Sainz de Baranda et al., 2010; Santonja et al., 2007) y el

Tabla 3. Características de la muestra en cada uno de los estudios seleccionados

\begin{tabular}{|c|c|c|c|c|}
\hline Estudio & Número & \multicolumn{2}{|c|}{ Sexo (chico o chica) } & Edad \\
\hline Coledam et al. (2012) & 58 & 29 & 29 & 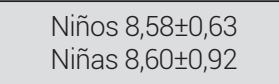 \\
\hline Martínez-García (2013) & 205 & 99 & 106 & $13,91 \pm 1,37$ \\
\hline Mayorga-Vega et al. (2014) & 73 & 36 & 37 & $9 \pm 0,2$ \\
\hline Merino-Marban et al. (2015) & 45 & - & - & 5,91 \\
\hline Rodríguez-García et al. (2008) & 90 & 41 & 49 & $\begin{array}{c}\text { E. Primaria } 10,27 \pm 0,31 \\
\text { E. Secundaria } \\
13,46 \pm 0,68\end{array}$ \\
\hline Rodríguez-García (2013) & 84 & - & - & $\begin{array}{c}\text { E. Primaria } 10,27 \pm 0,31 \\
\text { E. Secundaria } \\
13,46 \pm 0,68\end{array}$ \\
\hline Sainz de Baranda et al. (2006) & 62 & 31 & 31 & $\begin{array}{l}\text { G.E } 10,5 \pm 05 \\
\text { G.C } 10,3 \pm 0.3\end{array}$ \\
\hline Sainz de Baranda (2009) & 50 & 23 & 27 & $13,65 \pm 0,4$ \\
\hline Sainz de Baranda et al. (2010) & 80 & 43 & 37 & $\begin{array}{c}\text { Grupo } \\
\text { A: } 10,3 \pm 03 \\
\text { B: } 10,5 \pm 0,5 \\
\text { C: } 10,28 \pm 0,32\end{array}$ \\
\hline Sánchez-Rivas et al. (2014) & 44 & 20 & 24 & $7,84 \pm 0,37$ \\
\hline Santonja et al. (2014) & 62 & - & - & $\begin{array}{c}\text { Grupo } \\
\text { A: } 10,3 \pm 0,5 \\
\text { B: } 10,5 \pm 0,6 \\
\text { C: } 10,3 \pm 0,3\end{array}$ \\
\hline
\end{tabular}

Fuente: elaboración propia 
otro bloque formado por 4 estudios que emplearon un tiempo menor

Con relación al tiempo empleado para realizar el trabajo de flexibilidad, los estudios emplean un rango entre 1 y 7 minutos.

En cuanto a la técnica de estiramiento empleada, 7 de los estudios utilizan el estiramiento estático pasivo (Mayorga-Vega et al., 2014; Merino-Marban et al., 2015; Rodríguez-García et al., 2008; Rodríguez-García et al., 2013; Sainz de Baranda et al., 2006; Sánchez-Rivas et al., 2014; Santonja et al., 2007) 3 el estiramiento estático activo (Coledam et al., 2012; Martínez-García, 2013; Sainz de Baranda et al., 2010) y Sainz de Baranda (2009) que utiliza una combinación entre estiramiento estático activo y pasivo.

Con relación al método de medición de la extensibilidad isquiosural, 6 estudios utilizan el test dedos planta (Coledam et al., 2012; Mayorga-Vega et al., 2014; Merino-Marban et al., 2015; RodríguezGarcía et al., 2008; Sainz de Baranda et al., 2010; Sánchez-Rivas et al., 2014) 3 estudios utilizan el test de elevación de la pierna recta (MartínezGarcía, 2013; Sainz de Baranda, 2009; Santonja et al., 2007) y un estudio el test dedos suelo (Sainz de Baranda et al., 2006).

A continuación, por orden alfabético, se realiza un resumen de cada uno de los 11 artículos seleccionados.

\section{Coledam et al. (2012)}

En este trabajo se propusieron como objetivo medir el efecto crónico del estiramiento pasivo realizado durante el calentamiento sobre la flexibilidad del escolar. Para ello, llevaron a cabo un programa de estiramientos con 58 niños pertenecientes al municipio de Nova Europa (Sao Paulo, Brasil). El programa de intervención consistió en la realización de estiramientos durante las clases de Educación Física 2 veces a la semana. Se dividió a los participantes en dos grupos, grupo control y grupo experimental, y la extensibilidad isquiosural fue evaluada mediante el test dedos planta.

El grupo control no realizó estiramientos en su rutina de calentamiento. Por su parte, el grupo experimental realizaba 5 minutos de estiramientos como parte del calentamiento. Los estiramientos se realizaron mediante el método estático activo y sumaron un total de 6 ejercicios, de los cuales 2 eran específicos para la musculatura isquiosural.
Tras realizar la segunda medición, una vez finalizado el programa, los resultados mostraron como los chicos y chicas del grupo experimental habían mejorado su puntuación de forma significativa $(p<0,0001)$ en el test dedos planta (DDP) después de 16 semanas de intervención, siendo mayor la puntuación de las chicas que la de los chicos (17,94\% y 16,79\% respectivamente). Sin embargo, en el grupo control no se encontraron diferencias significativas entre el pre y post-test $(p>0,005)$ con un porcentaje de cambio de $-1,24 \%$ entre los chicos y un 1,29\% entre las chicas.

\section{Martínez-García (2013)}

Martínez-García (2013) se plantea el objetivo de determinar la influencia de un programa de Educación Postural sobre la disposición sagital estática y dinámica del raquis, la resistencia muscular lumbo-abdominal, la extensibilidad de la musculatura isquiosural y del psoas-iliaco, y las algias vertebrales en una muestra de escolares de Enseñanza Secundaria Obligatoria. Otro de los objetivos fue determinar los efectos diferenciales del programa en función del sexo y del ciclo formativo.

La muestra estudiada perteneció a 2 centros de Enseñanza Secundaria Obligatoria de la Región de Murcia. En total sumaron 205 alumnos que se distribuyeron en un grupo experimental $(n=117)$ y en un grupo control $(n=88)$. A su vez cada grupo fue estudiado según el ciclo al que pertenecían.

La duración del programa fue de 30 semanas y la frecuencia semanal fue de 1 sesión por semana. Se utilizaron 20 minutos de la clase habitual de Educación Física para desarrollar la intervención. Las actividades de trabajo consistieron en la realización de ejercicios de estiramiento, toma de conciencia postural y fortalecimiento muscular sobre diferentes grupos musculares.

Los ejercicios de estiramientos se mantuvieron invariables durante todo el curso a diferencia de los ejercicios de toma de conciencia y de fortalecimiento. Éstos se realizaron mediante la técnica de estiramiento estática y tuvieron una duración de entre 10 segundos en el primer trimestre, 12 segundos en el segundo trimestre y 15 segundos en el tercer trimestre. Los ejercicios de estiramiento de la musculatura isquiosural fueron 2 (1 ejercicio unilateral y 1 ejercicio bilateral) y se realizó una sola repetición por ejercicio.

Al principio y al final del programa se llevó a 
cabo una valoración del morfotipo sagital del raquis, la extensibilidad isquiosural y del psoas iliaco y la resistencia muscular de los extensores y flexores del tronco. Para valorar el raquis se midió el valor angular de las curvas sagitales con el inclinómetro. La flexibilidad isquiosural se determinó mediante el test de Elevación de la Pierna Recta (EPR); la flexibilidad del psoas ilíaco se valoró mediante el test de Thomas modificado. La resistencia muscular de los extensores del tronco se determinó mediante el Test de BieringSorensen y la resistencia muscular de los flexores del tronco mediante el Test Bench Trunk-Curl.

Tras analizar los datos obtenidos referentes a la extensibilidad de la musculatura isquiosural se aprecia un incremento de escolares con extensibilidad normal y un descenso de escolares con cortedad isquiosural de grado I. Encontrando diferencias significativas en los grupos experimentales $(p=, 0001)$ independientemente del ciclo $(p=, 913)$ y el sexo $(p=, 710)$.

\section{Mayorga-Vega et al. (2014)}

Mayorga-Vega et al. (2014) realizaron un estudio para comparar los efectos de un programa de estiramientos realizados en clase de Educación Física duranteel calentamiento o la vuelta a la calma sobre la flexibilidad en los escolares entre 9 y 10 años. El programa de intervención se llevó a cabo durante las clases de educación física. La duración del programa fue de 10 semanas y la frecuencia semanal de 2 sesiones por semana. La muestra seleccionada fueron 73 escolares, 36 chicos y 36 chicas. Los escolares quedaron divididos en 3 grupos según el momento de la sesión en el que realizaban los estiramientos: grupo control $(n=24)$, grupo de calentamiento $(n=25)$ y grupo de vuelta a la calma $(n=24)$.

El programa de estiramientos consistió en la ejecución de 6 ejercicios de estiramientos de la musculatura isquiosural en series de 20 segundos mediante la técnica estática pasiva. Para valorar la eficacia del programa se realizaron 2 medidas de la musculatura isquiosural mediante el test DDP. Se realizó antes de comenzar el programa (pretest) y una vez finalizado (post-test).

Los resultados muestran que los dos grupos experimentales presentan valores de extensibilidad isquiosural mayores que el grupo control, sin embargo, los resultados pre- y pos-test en estos grupos no son significativos respecto al aumento de la flexibilidad, aunque el grupo de estiramientos en la vuelta a la calma muestra valores ligeramente superiores al grupo de calentamiento.

\section{Merino-Marban et al. (2015)}

En comparación con los estudios anteriores que analizaban los efectos de los estiramientos durante el calentamiento o la vuelta a la calma, Merino-Marban et al. (2015) llevan a cabo un estudio con el objetivo de evaluar los efectos de un programa de estiramientos de 8 semanas de duración tras 5 semanas de desentrenamiento. La muestra estuvo compuesta por un total de 45 niños que se dividieron en dos grupos, grupo control y experimental. El grupo experimental realizó 1 minuto de estiramientos dos veces a la semana en la fase de vuelta a la calma de las clases de educación física. Los investigadores programaron 4 tipos de ejercicios que variaban cada semana.

La medición de la flexibilidad de la musculatura isquiosural se realizó mediante el test DDP en 3 momentos. Antes de comenzar el programa de intervención (pre-test), al finalizarlo (post-test) y tras 5 semanas de desentrenamiento (re-test). Tras analizar los datos obtenidos se observa que en el grupo experimental hay diferencias significativas entre el pre-test y pos-test $(p>0,001)$ sin embargo los niveles de flexibilidad descienden significativamente entre el post-test y el retest ( $p>0,001)$ sin llegarahaber diferencias significativas entre el pre-test y el retest $(p=1,000)$. Por otro lado, el grupo control no muestra diferencias significativas en ninguna medida $(p=1,000)$.

\section{Rodríguez-García et al. (2008)}

Rodríguez-García et al. (2008) centran el objetivo de su estudio en determinar si la realización de un programa de estiramientos realizado por escolares y adolescentes durante las clases de educación física puede mejorar la puntuación en el test DDP. El programa se desarrolló a lo largo de 32 semanas (un curso escolar), y las sesiones de estiramientos se realizaban 2 veces por semana.

La muestra sobre la que se llevó a cabo el estudio estaba compuesta por 46 escolares de Educación Primaria (20 chicos y 26 chicas) y 44 escolares de Educación Secundaria (21 chicos y 23 chicas). Éstos se dividieron en un grupo control que continuó las clases de educación física con 
normalidad, y un grupo experimental que realizó ejercicios de estiramientos de la musculatura isquiosural al final del calentamiento durante 3 minutos, y en la vuelta a la calma durante otros 2 minutos.

La evaluación de la flexibilidad de la musculatura isquiosural se realizó antes de iniciar el programa de intervención (pre-test) y al finalizarlo (posttest) mediante el test DDP. Los datos recogidos muestran que en el grupo de escolares de primaria hay una mejora leve y no significativa $(+1,95 \mathrm{~cm})$, al contrario que en el grupo de secundaria $(+7,22 \mathrm{~cm})$. Los grupos control de las dos etapas educativas mostraron una reducción de su puntuación en el test $(-4,38 \mathrm{~cm}$ en primaria y $-2,31 \mathrm{~cm}$ en secundaria) aunque solo fue significativa en el grupo de escolares de primaria.

\section{Rodríguez-García et al. (2013)}

Rodríguez-García et al. (2013) se plantean el objetivo de evaluar la eficacia de un programa sobre el dolor de espalda en escolares de Educación Primaria y Secundaria. La duración del programa fue de 32 semanas (un curso escolar) y la frecuencia semanal de 2 veces por semana. La muestra quedó compuesta por 41 alumnos de primaria $(n=41)$ y 43 estudiantes de secundaria (n=43). La edad media fue de 10,27 y 13,46 años respectivamente. El total de la muestra fue dividida en un grupo experimental y un grupo control.

En este programa se pidió la participación de los padres, ya que tenían que rellenar un cuestionario sobre la prevalencia de dolor lumbar de sus hijos antes (pre-test) y después (post-test) del programa. Los alumnos también rellenaron el mismo cuestionario en la escuela con la supervisión de sus profesores. Este cuestionario fue utilizado como instrumento de medida al finalizar el programa para evaluar si la prevalencia de dolor lumbar había descendido o aumentado.

El programa comenzó a la vez que el curso escolar y consistió en realizar un programa de actividad física que incluía 5 minutos de ejercicios de fuerza abdominal y lumbar, 3 minutos de estiramientos de la musculatura isquiosural y 5 minutos de ejercicios de concienciación pélvica. El grupo control no realizó este programa de actividad física.

Una vez finalizado el programa, los resultados muestran un descenso de la frecuencia del dolor lumbar en el grupo experimental y un incremento en el grupo control. En el pre-test el 9,5\% y el 11,9\% de los sujetos del grupo experimental y control respectivamente indicaron molestias en la zona lumbar. Tras el programa solo un 2,9\% de los sujetos del grupo experimental volvieron a indicar esas molestias mientras que en el grupo control aumentó hasta el 22,6\%.

\section{Sainz de Baranda et al. (2006)}

El siguiente estudio de Sainz de Baranda et al. (2006) se investiga sobre el efecto de un programa de estiramientos sobre la flexibilidad isquiosural en escolares prepúberes. Dicho programa se realizó durante 32 semanas, 2 sesiones por semana en escolares entre 9 y 10 años. El total de alumnos participantes fue de 63, y fueron asignados aleatoriamente al grupo control $(n=18)$ y al grupo experimental $(n=45)$.

El grupo experimental realizó el programa de estiramientos de la musculatura isquiosural. Los ejercicios se realizaron durante las clases de educación física, se destinaron 3 minutos del calentamiento y 2 minutos de la vuelta a la calma a estirar. Se diseñaron 4 tipos de ejercicios de estiramientos estático que alternaban entre la posición de bipedestación y de sedentación, y que variaban en cada sesión. Cada ejercicio fue ejecutado durante 20 segundos.

La medición de la flexibilidad isquiosural se realizó antes (pre-test) y después (post-test) del programa de intervención mediante el test dedos suelo (DDS). Los datos recogidos muestran una mejora significativa en el grupo experimental $(p<0,001)$ que pasa de una media de $21,8 \pm 5 \mathrm{~cm}$ a $29,8 \pm 5 \mathrm{~cm}$. Mientras que en el grupo control se observa una disminución pasando de un valor medio de $23,2 \pm 6 \mathrm{~cm}$ a $20,3 \pm 5,3 \mathrm{~cm}$, aunque las diferencias no llegan a ser significativas.

\section{Sainz de Baranda (2009)}

En el siguiente trabajo, Sainz de Baranda (2009) también evalúa el efecto de un programa de estiramientos sobre la extensibilidad de la musculatura isquiosural, aunque en este caso con escolares de Enseñanza Secundaria Obligatoria. La duración del programa de intervención fue de 31 semanas. Para el desarrollo de la investigación fue elaborado un diseño cuasiexperimental multigrupo, con grupo control y experimental con análisis de las medidas intragrupo e intergrupo. 
La muestra quedó formada por un total de 50 escolares (23 niños y 27 niñas) pertenecientes a 2 cursos de $2^{\circ}$ de la ESO. Los escolares se dividieron de forma natural debido a la aplicación del programa durante las clases de educación física, y se dividieron aleatoriamente en el grupo control y experimental, quedando un total de 24 alumnos en el grupo control y 26 en el grupo experimental.

El programa de intervención consistió en la realización de una rutina de estiramientos durante las clases de educación física. La técnica de estiramiento utilizada fue la técnica activa en el calentamiento y la técnica pasiva en la vuelta a la calma. Se realizaron 4 ejercicios en 5 minutos de calentamiento y 2 ejercicios en 2 minutos de vuelta a la calma. Se realizaron 3 repeticiones de 15 segundos por ejercicio con 5 segundos de descanso entre cada repetición.

La medición de la flexibilidad de la musculatura isquiosural se realizó mediante el test de EPR. Las mediciones se realizaron al principio (pre-test) y al final del programa (post-test). Una vez analizados los datos se observó una mejora significativa en el grupo experimental $(p<0,0001)$, con una ganancia de $+9,34^{\circ}$ entre el pre-test y el post-test. En el grupo control no se observaron cambios significativos $(p<0,05)$, de hecho se vio una reducción de la flexibilidad de $-2,21^{\circ}$ entre el pre-test y el post-test.

\section{Sainz de Baranda et al. (2010)}

En el estudio de Sainz de Baranda et al. (2010) analizaron el efecto de un programa de educación postural sobre el plano sagital de la columna vertebral. Diseñaron un programa de intervención de una duración de 9 meses (31 semanas) que coincidía con el curso escolar. La frecuencia semanal fue de 2 veces a la semana y el programa fue desarrollado dentro de las clases de educación física.

La muestra utilizada en la investigación fue seleccionada en un centro de enseñanza primaria de la Región de Murcia, y quedó formada por 80 escolares (43 niños y 37 niñas) de 3 clases diferentes de $5^{\circ}$ curso. Se distribuyeron de forma natural a la hora de recibir las sesiones y de forma aleatoria en el grupo control y experimental, quedando un total de 3 grupos: grupo experimental 1 de $5^{\circ} \mathrm{A}$; grupo experimental 2 de $5^{\circ} \mathrm{B}$; y grupo control de $5^{\circ} \mathrm{C}$.

El programa estaba compuesto por 3 bloques: ejercicios de toma de conciencia y percepción pélvica (bloque 1), ejercicios de fortalecimiento abdominal y lumbar (bloque 2), y ejercicios de estiramientos de la musculatura isquiosural (bloque 3). Este último bloque estuvo compuesto por estiramientos realizados mediante la técnica activa manteniendo siempre la columna vertebral alineadaycon unaanteversiónpélvica. Serealizaron 4 ejercicios en 5 minutos de calentamiento y 2 ejercicios en 2 minutos de calentamiento. De cada ejercicio se realizaron 3 repeticiones de 15 segundos por ejercicio.

Para evaluar el efecto del programa se establecieron una serie de pruebas antes y después de la aplicación de éste. Se midió la curva dorsal y lumbar del raquis en bipedestación habitual, sedentación asténica y flexión máxima del tronco. Una vez tomados todos los datos se observan mejoras significativas en los dos grupos experimentales en todas las valoraciones excepto en la cifosis y lordosis en bipedestación $(p<0,001)$. En el grupo control no se observó ninguna mejora, de hecho, empeoró la disposición sagital estática en bipedestación y dinámica en flexión de tronco y sedentación.

\section{Sánchez-Rivas et al. (2014)}

Sánchez-Rivas et al. (2014) analizan el efecto de un programa de estiramientos de 3 minutos de duración sobre la musculatura isquiosural realizado durante las clases de educación física. La muestra sobre la que se aplicó el programa estuvo constituida por 44 escolares (24 niñas y 20 niños) de tercer curso de primaria. La división por grupos fue natural ya que cada sujeto realizaba la sesión durante su clase de educación física, pero la distribución en grupo control y experimental fue de forma aleatoria quedando 22 alumnos en el grupo control y 22 en el experimental.

La duración del programa fue de 9 semanas y la frecuencia semanal de 2 sesiones por semana. El programa consistió en la ejecución de estiramientos de la musculatura isquiosural durante 3 minutos a final de la vuelta a la calma de la sesión. Se realizaron 3 ejercicios bilaterales en sedentación y en bipedestación mediante la técnica de estiramiento estática pasiva.

Una semana antes de comenzar el programa evaluaron la extensibilidad isquiosural mediante el test DDP (pre-test) y lo volvieron a medir una vez finalizado. Los datos muestran pequeñas diferencias al comparar el grupo control y el 
experimental, a favor de éste último pues se observó una mejora de $1,1 \pm 2,7 \mathrm{~cm}$, mientras que el grupo control se observó una reducción de la flexibilidad de $0,6 \pm 1,1 \mathrm{~cm}$. Sin embargo, el tamaño del efecto del programa de flexibilidad fue moderado-bajo $(g=0,37)$.

\section{Santonja et al. (2007)}

Santonja et al. (2007) realizaron un estudio en el que evaluaron la eficacia de diferentes frecuencias de un programa de ejercicios de estiramientos sobre el rango de movimiento articular (ROM) de la extremidad inferior en 62 escolares prepúberes (10-11 años). La duración del programa fue de 31 semanas.

Los cursos fueron seleccionados aleatoriamente para ser grupo control o grupo experimental. El grupo experimental A estaba formado por 25 sujetos y realizaron 5 minutos de estiramientos en las clases de educación física 2 veces a la semana. Por otro lado, el grupo experimental B, formado por 20 sujetos realizaron 5 minutos de estiramientos en las clases de educación física y en las actividades extraescolares, contabilizando un total de 4 sesiones por semana. El grupo control estuvo constituido por 18 escolares que continuaron con las clases normales de educación física.

Los grupos experimentales realizaron 3 minutos de estiramientos de la musculatura isquiosural en el calentamiento y otros 2 minutos en la vuelta a la calma. Se seleccionaron 4 ejercicios que fueron variando en cada semana.

Para medir la flexibilidad de la musculatura isquiosural se utilizó el test EPR con ambas piernas. Se tomaron medidas antes de comenzar con el programa (pre-test) y al finalizarlo (post-test). Los resultados obtenidos no muestran diferencias significativas entre la flexibilidad isquiosural de la pierna derecha y la izquierda, pero si muestran mejoras significativas entre el pre-test y el post-test en los 2 grupos experimentales $(p<0,001)$ siendo el grupo $\mathrm{B}$ el que mayores ganancias presentó $\left(+16,92^{\circ}\right)$. Sin embargo el grupo control redujo la flexibilidad en $-3,1^{\circ}$

\section{Discusión}

El objetivo de este trabajo de revisión ha sido valorar la eficacia de los programas de educación postural que han sido llevados a cabo en el contexto escolar y que han introducido estiramientos específicos de la musculatura isquiosural.
Al analizar la duración de los programas se ha encontrado un rango entre las 8 y 32 semanas. Un mayor número de estudios utilizan 32 semanas, y sólo 4 de los 11 estudios emplean entre 8 y 16 semanas para aplicar el programa. Los resultados indican que las mejoras significativas en los valores de flexibilidad se obtienen en los programas de mayor duración. Destacando el estudio de Rodríguez-García et al. (2008) y Santonja et al. (2007) como los que muestran mayores ganancias en flexibilidad. Por el contrario, Sánchez-Rivas et al. (2014) y Mayorga-Vega et al. (2014) no encuentran diferencias significas entre las medidas de cada grupo (tabla 4).

Con relación a la frecuencia semanal de las sesiones destacar que todos los programas utilizan las 2 sesiones semanales de las clases de Educación Física excepto Martínez-García (2013) que solo utiliza un día de la semana. Son numerosos los programas que aplican ejercicios de estiramientos de flexibilidad isquiosural durante las clases de educación física 2 veces a la semana y muestran importantes mejoras de la flexibilidad isquiosural (Rodríguez et al. 2008; Sainz de Baranda et al., 2006).

En cuanto a la duración del tiempo específico empleado para estirar la musculatura isquiosural se observa un tiempo de aplicación entre 1 y 8 minutos.

Con relación a la duración aislada del estiramiento todos los programas utilizan 20 segundos (Coledam et al., 2012; Mayorga-Vega et al., 2014; Merino-Marban et al., 2015; RodríguezGarcía et al., 2008; Rodríguez-García et al., 2013; Sainz de Baranda 2006; Sánchez-Rivas et al., 2014; Santonja et al., 2007) a excepción de MartínezGarcía (2013), Sainz de Baranda (2009) y Sainz de Baranda (2010) que establecen como duración aislada de los estiramientos los 15 segundos.

Son muchos los autores los que han investigado sobre la duración aislada del estiramiento, sin embargo, no hay una evidencia clara sobre qué duración del estiramiento es la más adecuada (Ayala y Sainz de Baranda, 2008).

Bandy e Irion (1994) señalan, tras comparar la eficacia de estirar de forma pasiva durante 15, 30 y 60 segundos, que estirar 30 segundos era la duración más eficaz.

Roberts y Wilson (1999) señalan que es igual de efectivo realizar en un estiramiento pasivo 9 repeticiones de 5 segundos o 3 repeticiones de 15 segundos. 
Tabla 4. Efectos del programa de estiramientos sobre la extensibilidad isquiosural en los estudios analizados

\begin{tabular}{|c|c|c|c|c|c|}
\hline \multirow[t]{2}{*}{ Estudio } & \multirow[t]{2}{*}{ Grupo } & \multicolumn{2}{|c|}{ Momento de la medida } & \multirow[t]{2}{*}{ Diferencia } & \multirow[t]{2}{*}{ Re-test } \\
\hline & & Pre & Post & & \\
\hline Coledam et al. (2012) & $\begin{array}{l}\text { HGC } \\
\text { HGE* } \\
\text { MGC } \\
\text { MGE* }\end{array}$ & $\begin{array}{l}24,17 \mathrm{~cm} \\
24,89 \mathrm{~cm} \\
28,60 \mathrm{~cm} \\
27,25 \mathrm{~cm}\end{array}$ & $\begin{array}{l}23,87 \mathrm{~cm} \\
29,07 \mathrm{~cm} \\
28,97 \mathrm{~cm} \\
34,12 \mathrm{~cm}\end{array}$ & $\begin{array}{c}-0,3 \mathrm{~cm} \\
4,18 \mathrm{~cm} \\
-0,37 \mathrm{~cm} \\
6,87 \mathrm{~cm}\end{array}$ & \\
\hline Martínez-García (2013) & $\begin{array}{l}\text { GC* } \\
G E *\end{array}$ & $\begin{array}{l}78,4^{\circ} \\
81,6^{\circ}\end{array}$ & $\begin{array}{l}77,2^{\circ} \\
87,9^{\circ}\end{array}$ & $\begin{array}{r}-1,2^{\circ} \\
6,3^{\circ}\end{array}$ & \\
\hline Mayorga-Vega et al. (2014) & $\begin{array}{c}\text { GC } \\
\text { Calentamiento } \\
\text { Vuelta a la calma }\end{array}$ & $\begin{array}{l}12,6 \mathrm{~cm} \\
10,2 \mathrm{~cm} \\
11,8 \mathrm{~cm}\end{array}$ & $\begin{array}{c}9,9 \mathrm{~cm} \\
9,9 \mathrm{~cm} \\
12,3 \mathrm{~cm}\end{array}$ & $\begin{array}{c}-2,8 \mathrm{~cm} \\
-0,3 \mathrm{~cm} \\
0,5 \mathrm{~cm}\end{array}$ & \\
\hline Merino-Marban et al. (2015) & $\begin{array}{l}\mathrm{GC} \\
\mathrm{GE}\end{array}$ & $\begin{array}{l}16,9 \mathrm{~cm} \\
16,4 \mathrm{~cm}\end{array}$ & $\begin{array}{l}16,9 \mathrm{~cm} \\
18,8 \mathrm{~cm}\end{array}$ & $\begin{array}{c}0 \mathrm{~cm} \\
2,4 \mathrm{~cm}\end{array}$ & $\begin{array}{l}16,9 \mathrm{~cm} \\
16,6 \mathrm{~cm}\end{array}$ \\
\hline Rodríguez-García et al. (2008) & $\begin{array}{l}\text { GC Primaria* } \\
\text { GC Secundaria* } \\
\text { GE Primaria* } \\
\text { GE Secundaria* }\end{array}$ & $\begin{array}{l}0,44 \mathrm{~cm} \\
-0,38 \mathrm{~cm} \\
-0,65 \mathrm{~cm} \\
-4,00 \mathrm{~cm}\end{array}$ & $\begin{array}{c}-3,94 \mathrm{~cm} \\
-2,69 \mathrm{~cm} \\
1,30 \mathrm{~cm} \\
+3,22 \mathrm{~cm}\end{array}$ & $\begin{array}{l}-4,38 \mathrm{~cm} \\
-2,31 \mathrm{~cm} \\
+1,95 \mathrm{~cm} \\
+7,22 \mathrm{~cm}\end{array}$ & \\
\hline Sainz de Baranda et al. (2006) & $\begin{array}{l}\text { GC } \\
\text { GE* }\end{array}$ & & & $\begin{array}{l}-2,9 \mathrm{~cm} \\
+8 \mathrm{~cm}\end{array}$ & \\
\hline Sainz de Baranda (2009) & $\begin{array}{l}\mathrm{GC} \\
\mathrm{GE}\end{array}$ & $\begin{array}{l}79,2^{\circ} \\
79,7^{\circ}\end{array}$ & $\begin{array}{l}77,3^{\circ} \\
87,3^{\circ}\end{array}$ & $\begin{array}{l}-2,21^{\circ} \\
9,345^{\circ}\end{array}$ & \\
\hline Sánchez-Rivas et al. (2014) & $\begin{array}{l}\text { GC } \\
\text { GE }\end{array}$ & $\begin{array}{l}16,6 \mathrm{~cm} \\
17,1 \mathrm{~cm}\end{array}$ & $\begin{array}{c}16 \mathrm{~cm} \\
18,2 \mathrm{~cm}\end{array}$ & $\begin{array}{l}-0,6 \mathrm{~cm} \\
1,1 \mathrm{~cm}\end{array}$ & \\
\hline Santonja et al. (2007) & $\begin{array}{l}\text { GC* } \\
\text { GEA* } \\
\text { GEB* }\end{array}$ & $\begin{array}{l}79,2^{\circ} \\
77,7^{\circ} \\
76,7^{\circ}\end{array}$ & $\begin{array}{l}77,7^{\circ} \\
86,7^{\circ} \\
93,7^{\circ}\end{array}$ & $\begin{array}{c}-3,111^{\circ} \\
9,345^{\circ} \\
16,92^{\circ}\end{array}$ & \\
\hline
\end{tabular}

* Resultados estadísticamente significativos

HCG: Hombres grupo control; HGC: Hombres grupo experimental; MGC: mujeres grupo control; MGE: Mujeres grupo experimental; GC: grupo control; GE: grupo experimental

Por su parte, Ford, Mazzone y Taylor (2005) no encontraron diferencias significativas al analizar 4 duraciones diferentes (30, 60, 90 y 120 segundos) del estiramiento estático-activo.

Ayala y Sainz de Baranda (2008) compararon la eficacia de realizar 6 series de 30 segundos y 12 series de 15 segundos, llegando a la conclusión de que ambas duraciones son igual de efectivas, pues la clave de la eficacia reside en la duración total de la sesión de estiramientos (en este estudio 180 segundos) y no en la duración aislada del estiramiento.

Con relación a la técnica de estiramientos, los diferentes estudios utilizan la técnica estática pasiva (Mayorga-Vega et al., 2014; MerinoMarban et al., 2015; Rodríguez-García et al., 2008; Rodríguez-García et al., 2013; Sánchez-Rivas et al., 2014; Santonja et al., 2007) o la técnica estática activa (Coledam et al., 2012; Martínez-García, 2013; Sainz de Baranda, 2010). Solamente Sainz de Baranda (2009) realiza una combinación de ambas técnicas de estiramiento asociando cada técnica al calentamiento o a la vuelta a la calma.

La utilización de la técnica activa mediante la realización de una anteversión pélvica durante el estiramiento, es un método muy eficaz para aumentar la flexibilidad de la musculatura isquiosural (Ford et al., 2005; Sullivan, Dejulia y Worrell 1992) además de que cumple con criterio de seguridad para la columna vertebral debido al mantenimiento de la lordosis lumbar dentro de los valores de normalidad (McGill, 2007; Kolber y Zepeda 2004; Sullivan et al., 1992).

Por otro lado, la eficacia de la técnica de estiramiento pasivahasidoprobada pornumerosos estudios (Ayala y Sainz de Baranda, 2008). Spring (1998) señala que el estiramiento pasivo es el más adecuado para realizar en los programas de acondicionamiento físico y gimnasia en general, siendo una técnica que asegura las condiciones de elongación dentro de la normalidad. Dubreuil y Neiger (1995) destacan que ésta técnica es adecuada cuando existe limitación de la movilidad articular y acortamiento muscular que dificulta la realización de cualquier gesto.

Otro aspecto a analizar es el momento de la sesión de aplicación del estiramiento. Algunos estudios utilizan el calentamiento para la realización de los estiramientos (Coledam et al., 2012; Martínez-García, 2013), otros utilizan la 
vuelta a la calma (Sánchez-Rivas et al., 2014), Por su parte, Rodríguez-García et al. (2008), Sainz de Baranda et al. (2006), Sainz de Baranda, (2009), Sainz de Baranda et al. (2010) y Santonja et al. (2007) combinan ambos momentos realizando entre 3 y 5 minutos de estiramientos en el calentamiento y 2 minutos en la vuelta a la calma.

En esta variable cabe destacar el estudio de Mayorga-Vega et al. (2014) ya que comparan la diferencia entre estirar durante el calentamiento o la vuelta a la calma. Los resultados demuestran que en ambos grupos se producen los mismos resultados independientemente del momento de la sesión donde se realicen los estiramientos.

La extensibilidad de la musculatura isquiosural ha sido evaluada en la mayoría de los estudios mediante el test DDP. Mientras que otros estudios han utilizado el test de elevación de la pierna recta (EPR) o el test DDS.

El test DDP ha demostrado tener de forma generalizada una elevada fiabilidad relativa en sus resultados cuando se utiliza con escolares y adulos jóvenes sanos, independientemente del sexo (Ayala, Sainz de Baranda, de Ste Croix y Santonja, 2012).

Por otro lado, el test EPR es un test angular muy utilizado en elámbito clínico y científico como criterio de referencia de la extensibilidad isquiosural debido principalmente a su procedimiento exploratorio que solo implica el movimiento de una articulación (Ayala et al. 2012).

El test DDS es una alternativa válida como el test DDP como estimación de la extensibilidad is $\neg$ quiosural, con valores de correlación respecto al test EPR ligeramente superiores al DDP (RodríguezGarcía et al., 2008). Una posible explicación a los mayores niveles de correla-ción mostrados por el DDS en comparación al DDP pueda radicar en que: a) al realizar el DDS el tronco sobrepasa la horizontal gracias a un movi-miento de flexión de la pelvis, con lo que se logra mayor alcance con menor flexión vertebral (Kippers y Parker, 1984); b) en el DDS hay menor limitación de movi-miento de la pelvis al no estar apoyada en el suelo y además el papel de la fuerza de la gravedad es mayor (Liemohn, Sharpe y Wasserman, 1994).

Sainz de Baranda etal. (2006) sugieren la utilización del test DDS en las exploraciones clínicas debido a que los movimientos de flexión de tronco en bipedestación son gestos muy utilizados, tanto en las actividades de la vida diaria como en muchos gestos deportivos.

\section{Conclusiones}

- Tras analizar la duración de los programas de estiramiento se ha encontrado un rango entre la 8 y las 32 semanas. Un mayor número de estudios utilizan 32 semanas, y sólo 4 de los 11 estudios emplean entre 8 y 16 semanas para aplicar el programa. Los resultados indican que las mejoras significativas en los valores de flexibilidad se obtienen en los programas de mayor duración.

- Ante una actitud no intervencionista los valores de flexibilidad no mejoran e incluso empeoran. Estos datos muestran la importancia de introducir la realización sistemática de estiramientos dentro de las clases de Educación Física.

- La frecuencia más utilizada para realizar un programa de estiramientos es de 2 días a la semana aprovechando normalmente las clases de Educación Física. Aunque, realizar los estiramientos durante 1 día a la semana también produce cambios significativos en la extensibilidad isquiosural.

- Con relación a la duración aislada del estiramiento el $72,8 \%$ de los programas utilizan 20 segundos y el 27,2\% establecen la duración de 15 segundos. - El momento de aplicación de los estiramientos en la sesión puede variar entre el principio de la sesión, la vuelta a la calma o una combinación de ambos, siendo los 3 momentos igual de efectivos.

\section{Aplicaciones prácticas}

Los resultados extraídos deben servir para reflexionar sobre los efectos del trabajo de flexibilidad en la edad escolar y su inclusión en las clases de Educación Física.

Los resultados del estudio reflejan que la puesta en práctica de un programa de estiramientos puede ayudar a mantener y/o mejorar la extensibilidad de la musculatura isquiosural.

El tratamiento de la Educación Postural y el cuidado de la espalda dentro del área de Educación Física están suficientemente justificado y respaldado por el currículo educativo. Debido a ello, la Educación Postural, el ejercicio físico específico para el cuidado de la espalda y la higiene postural deben ser desarrollados y evaluados dentro de la materia de Educación Física. 


\section{Referencias}

Andújar, P. (2010). Prevalencia de las desalineaciones sagitales del raquis en el Municipio de Murcia. [Tesis Doctoral]. Murcia: Universidad de Murcia.

Andújar, O., Alonso, C. \& Santonja, F. (1996). Tratamiento de la cortedad de isquiosurales. Selección, 5(1), 37-48.

Ayala, F., Sainz de Baranda, P. S., de Ste Croix, M. \& Santonja, F. (2012). Fiabilidad y validez de las pruebas sit-and-reach: revisión sistemática. Revista Andaluza de Medicina del Deporte, 5(2), 57-66.

Ayala, F. \& Sainz de Baranda, P. (2008). Efecto de la duración y técnica de estiramiento de la musculatura isquiosural sobre la flexión de cadera). Cultura Ciencia y Deporte, 3(8), 93-99.

Balagué, F., Troussier, B. \& Salminen, J.J. (1999). Non specific low back pain in children and adolescents: risk factors. European Spine Journal, 8(6), 429-438.

Bandy, W. D. \& Irion, J. M. (1994). The effect of time on static stretch on the flexibility of the hamstring muscles. Physical Therapy, 74(9), 845-850.

Cardoso, C. \& Gómez, A. (2008). Lumbalgia. Prevalencia y programas preventivos en la infancia y adolescencia. Revista iberoamericana de Fisioterapia y Kinesología, 11(1), 32-38.

Cardon, G. M., de Clercq, D. L., Geldhof, E. J., Verstraete, S. \& de Bourdeaudhuij, I. M. (2007). Back education in elementary schoolchildren: the effects of adding a physical activity promotion program to a back care program. European Spine Journal, 16(1), 125-133.

Coledam, D. H. C., Arruda, G. A. D. \& Oliveira, A. R. D. (2012). Chronic effect of static stretching performed during warm-up on flexibility in children. Revista Brasileira de Cineantropometria \& Desempenho Humano, 14(3), 296-304

Dubreuil, C. \& Neiger, H. (1984). Comparaison des effects de la course et des étirements autopassifs sur lextensibilité des ischio-jambiers. Annales de Kinésithérapie, 11(5), 191-195.

Ekstrand. J. \& Guillquist, J. (1982). The frequency of muscle tightness and injuries in soccer players. American Journal of Sport Medicine, 10(2), 75-78.

Feldman, D.E., Shrier, I., Rossignol, M. \& Abenhaim, L. (2002). Work is a risk factor for adolescent musculoskeletal pain. Journal of Occupational and Environmental Medicine, 44, 956-961.

Ferrer, V. (1998). Repercusiones de la cortedad isquiosural sobre la pelvis y el raquis lumbar. [Tesis Doctoral]. Universidad de Murcia.

Fernández, M.J. (2011). Efecto de un programa de Educación Postural en Educación Física en Primaria: tres años de seguimiento. [Tesis Doctoral]. Murcia: Universidad de Murcia.

Fernandes, S. M. S., Casarotto, R. A. \& João, S. M. A. (2008). Effects of educational sessions on school backpack use among elementary school students. Brazilian Journal of Physical Therapy, 12(6), 447-453.

Foltran, F. A., Moreira, R. F., Komatsu, M. O., Falconi, M. F. \& Sato, T. O. (2012). Effects of an educational back care program on Brazilian schoolchildren's knowledge regarding back pain prevention. Brazilian Journal of Physical Therapy, 16(2), 128133.

Ford, G.S., Mazzone, M.A. \& Taylos, K. (2005). The effect of 4 ifferent durations of static hamstring stretching on passive kneeextension range of motion. Journal of Sport Rehabilitation, 14 95-107.

García-Alen Lores, C. (2009). La educación postural en el ámbito educativo y su contexto social. Recuperado de: http://www. eduinnova.es/monografias09/EDUCACION_POSTURAL.pdf

Geldhof, E., Cardon, G., De Bourdeaudhuij, I., Danneels, L., Coorevits, P., Vanderstraeten, G. \& De Clercq, D. (2007). Effects of back posture education on elementary schoolchildren's back function. European Spine Journal, 16(6), 829-839.

Geldhof, E., Cardon, G., De Bourdeaudhuij, I. \& De Clercq, D. (2007).
Back posture education in elementary schoolchildren: a 2-year follow-up study. European Spine Journal, 16(6), 841-850.

Geldhof, E., Cardon, G., De Bourdeaudhuij, I. \& De Clercq, D. (2006). Effects of a two-school-year multifactorial back education program in elementary schoolchildren. Spine, 31(17), 19651973.

Grimmer, K., Dansie, B., Milanese, S., Pirunsan, U. \& Trott, P. (2002). Adolescent standing postural response to backpack loads: a randomized experimental study. Biomedical Central Musculoskeletal Disorders, 3, 10

Groll, C., Heine-Goldammer, B. \& Zalpour, C. (2009). Evaluation of a prevention concept in the primary school setting: Prevent back pain with "The Rücken Kult-Tour". Pravention und Gesundheitsforderung, 4(1), 51-57

Gunzbburg, R., Balagué, F., Nordin, M., Szpalski, M., Duyck, D., Bull, D. \& Melot, C. (1999). Low back pain in a population of school children. European Spine Journal, 8(6) 439-443.

Harreby, M., Neergaard, K., Jessen, T., Larsen, E., Storr-Paulsen, A., Lindahl, A., Fisker, I. \& Laegaard, E. (1999). Risk factors for low back pain in a cohort of 1389 Danish school children an epidemiologic study. European Spine Journal, 8(6), 444-450.

Hellsing, A.L. (1998). Tightness of hamstring and psoas major muscles. A prospective study of back pain in young men during their military service. Upsala Journal of Medical Sciences, 93(3), 267-276

Jones, G.T., Watson, K.D., Silman, A.J., Symmons, D.P.M. \& Macfarlane, G.J. (2003). Predictors of low back pain in British schoolchildren a population-based prospective cohort study. Pediatrics, $111(4 \mathrm{Pt} 1), 822-828$

Kolber, M. J. \& Zepeda, J. (2004). Addressing hamstring flexibility in athletes with lower back pain: A discussion of commonly prescribed stretching exercises. Strength \& Conditioning Journal, 26(1), 18-23

Liemohn, W., Sharpe, G. L. \& Wasserman, J. F. (1994). Criterion Related Validity of the Sit-and-Reach Test. The Journal of Strength \& Conditioning Research, 8(2), 91-94.

Lorenzo González, M. E. \& García Soidán, J. L. (2009). Elaboración y aplicación de un programa de educación postural en Educación Secundaria. Revista de Investigación en Educación, 2, 63-88.

Martínez García, A.C (2013). Efectos de un programa de Educación Postural sobre el Morfotipo Sagital del Raquis, la Extensibilidad de la Musculatura Isquiosural y el Psoas Iliaco y la Resistencia Muscular, Abdominal y Lumbar en Escolares en Educación Secundaria. Universidad de Murcia, Facultad de Medicina.

Martínez González, M., Gómez Conesa, A. \& Hidalgo Montesinos, M.D. (2008). Programas de higiene postural desarrollados con escolares. Fisioterapia, 30(5), 233-230

Mayorga-Vega, D., Merino-Marban, R., Garrido, F. J. \& Viciana, J. (2014). Comparison between warm-up and cool-down stretching programs on hamstring extensibility gains in primary schoolchildren. Physical Activity Review, 2, 16-24.

McGill, S.M. (2007). Low back disorders. Evidence-Based prevention and rehabilitation. Human kinetics. Champaign, Illinois.

Méndez, F. J. \& Gómez-Conesa, A. (2001). Postural hygiene program to prevent low back pain. Spine, 26(11), 1280-1286.

Merino-Marban, R., Mayorga-Vega, D., Fernandez-Rodriguez, E. Estrada, F. V. \& Viciana, J. (2015). Effect of a physical educationbased stretching programme on sit-and-reach score and its posterior reduction in elementary schoolchildren. European Physical Education Review, 21(1), 83-92.

Mesa, M. (1992). Diagnóstico diferencial del síndrome de isquiosurales cortos. I Jornadas de actualización del Centro de Medicina del Deporte "Síndrome de acortamiento de la musculatura isquiosural. Murcia.

Moore, M.J., White, G.L. \& Moore, D. (2007). Association of relative backpack weight with reported pain, pain sites, medical utilization, and lost school time in children and adolescents. Journal of School Health, 77(5), 232-239. 
Nelson, R. T. \& Bandy, W. D. (2004). Eccentric training and static stretching improve hamstring flexibility of high school males. Journal of Athletic Training, 39(3), 254-258.

Peña, W.A. (2010) Educación Física y Salud: Programa para la mejora del raquis en el plano sagital y la extensibilidad Isquiosural en Secundaria y Bachillerato. [Tesis Doctoral]. Murcia: Universidad de Murcia.

Rivas, A. (2015). Efectos de la unidad didáctica "Aprende a cuidar tu espalda" en alumnos de Educación Secundaria. [Tesis Doctoral]. Toledo: Universidad de Castilla La Mancha.

Real Decreto-ley 291/2007, de 14 de septiembre, por el que se establece el currículo de Educación Secundaria Obligatoria en la Comunidad Autónoma de la Región de Murcia. Boletín Oficial de la Región de Murcia, 24 de septiembre de 2007, núm. 221, pp. 27179-27303

Roberts, J. M. \& Wilson, K. (1999). Effect of stretching duration on active and passive range of motion in the lower extremity. British Journal of Sports Medicine, 33(4), 259-263.

Rodríguez-García, P. L., López-Miñarro, P. A. \& Santonja, F. M. (2013). The effect of school physical education programmes on low-back pain in schoolchildren. Journal of Physical Education \& Health, 4(2), 43-48.

Rodríguez, P. L., Santonja, F. M., López-Miñarro, P. A., Sainz de Baranda, P. \& Yuste, J. L. (2008). Effect of physical education stretching programme on sit-and-reach score in schoolchildren. Science \& Sports, 23(3), 170-175.

Rodríguez, P.L. (1998). Educación Física y salud del escolar: programa para la mejora de la extensibilidad isquiosural y del raquis en el plano sagital. [Tesis Doctoral]. Granada: Universidad de Granada.

Sainz de Baranda, P., García, P. L. R. \& Medina, F. S. (2010). Efectos sobre la disposición sagital del raquis de un programa de Educación Postural en Educación Física de Primaria. Apunts. Educación Física y Deportes, 102, 16-21.

Sainz de Baranda, P. (2009). El trabajo de la flexibilidad en educación física: Programa de intervención. Cultura Ciencia y Deporte, 4(10), 33-38

Sainz de Baranda, P., Rodríguez, P.L., Santonja, F. \& Andújar, P. (2006). La columna vertebral del escolar. Sevilla: Wanceulen.

Sainz de Baranda, P. (2002). Educación Física y Actividad Extraescolar: programa para la mejora del raquis en el plano sagital y la extensibilidad isquiosural en Primaria. [Tesis doctoral]. Murcia: Universidad de Murcia.

Sainz de Baranda, P., Rodríguez, P. L., Santonja, F., López, P. A., Andújar, P., Ferrer, V. \& Pastor, A. (2006). Effects of hamstring stretching exercises on the toe-touch test in elementary schoolchildren. Journal of Human Movement Studies, 51(4), 277-289.

Sánchez Rivas, E.; Mayorga-Vega, D.; Fernández Rodríguez, E. \& Merino-Marbán, R. (2014). Efecto de un programa de estiramiento de la musculatura isquiosural en las clases de educación física en Educación Primaria. Journal of Sport and Health Research, 6(2), 159-168.

Santonja, M. F., Sainz de Baranda, P., Rodríguez, G. P., López, M. P. \& Canteras, J. M. (2007). Effects of frequency of static stretching on straight-leg raise in elementary school children. The Journal of Sports Medicine and Physical Fitness, 47(3), 304-308.

Santonja Medina, F., Rodríguez García, P.L., Saiz de Baranda Andújar, P. \& López Miñarro, P.A. (2004). Papel del profesor de educación física ante las desalineaciones de la columna vertebral. Selección, 13(1), 5-17

Santonja, F., Ferrer V. \& Contreras. (1995). Exploración Clínica del Síndrome de isquiosurales cortos en el ámbito escolar. Revista Selección, 14(2), 81-91.

Santonja, F. \& Genovés, J.L. (1992). Radiología: Consideraciones en Ortopedia. En F. Santonja y I. Martínez (Eds.), Valoración médico-deportiva del escolar (pp. 279-301). Murcia: Universidad de Murcia.

Sheldon, M. R. (1994). Lifting instruction to children in an elementary school. Journal of Orthopaedic \& Sports Physical Therapy, 19(2), 105-110.

Sjölie, A.N. \& Ljunggren, A.E. (2001). The significance of high lumbar mobility and low lumbar strength for current and future low back pain in adolescents. Spine, 26(23), 2629-2636.

Sping, H. (1988). Qu' apporte l'streching? Annales de kinesitherapie, 15(1-2), 41-43

Sullivan, M. K., Dejulia, J. J. \& Worrell, T. W. (1992). Effect of pelvic position and stretching method on hamstring muscle flexibility. Medicine \& Science in Sports \& Exercise, 24(12), 1383-1389.

Vidal, J., Borrás, P. A., Cantallops, J., Ponseti, X. \& Palou, P. (2010). Propuesta de intervención para la prevención del dolor de espalda en el ámbito escolar. Revista de Transmisión del Conocimiento Educativo y de la Salud, 2(6), 536-551.

Wedderkopp, N., Kjaer, P., Hestbaek, L., Korsholm, L. \& Leboeuf-Yde, C. (2009). High-level physical activity in childhood seems to protect against low back pain in early adolescence. Spine, 9(2), 134-141.

Zakas, A., Galazoulas, C., Grammatikopoulou, M. G. \& Vergou, A. (2002). Effects of stretching exercise during strength training in prepubertal, pubertal and adolescent boys. Journal of Bodywork and Movement Therapies, 6(3), 170-176. 